\title{
Do inventory management practices affect economic performance? An empirical evaluation of the machine tool SMEs in Bangalore
}

\author{
Rajeev N* \\ Department of Management Studies, Indian Institute of Science, Bangalore- 560012, India
}

(Received December 23 2007, Accepted March 12 2008)

\begin{abstract}
Inventory management (IM) have a decisive role in the enhancement of manufacturing industry's competitiveness. Therefore, major manufacturing industries are following IM practices with the intention of improving their performance. However, the effort to introduce IM in SMEs is very limited due to lack of initiation, expertise, and financial constraints. This paper aims to provide a guideline for entrepreneurs in enhancing their IM performance, as it presents the results of a survey based study carried out for machine tool Small and Medium Enterprises (SMEs) in Bangalore. Having established the significance of inventory as an input, we probed the relationship between IM performance and economic performance of these SMEs. To the extent possible all the factors of production and performance indicators were deliberately considered in pure economic terms. All economic performance indicators adopted seem to have a positive and significant association with IM performance in SMEs. On the whole, we found that SMEs which are IM efficient are likely to perform better on the economic front also and experience higher returns to scale.
\end{abstract}

Keywords: economic performance, inventory management, SMEs

\section{Introduction}

Industrial surveys conducted in developing countries have shown that proper IM has a significant role to play in promoting industrial development ${ }^{[3]}$. In this paper, we intend to analyze IM performance from an economic point of view through production function analysis. We seek to establish the importance of inventory amongst all the inputs by estimating Cobb-Douglas production function in each of the three groups (performing, moderately performing and nonperforming respectively) of SMEs. Having established the significance of inventory as an input, we probe whether IM is associated with economic performance of SMEs in the machine tool sector. The IM efficiency group based analysis suggested a strong relationship between IM and economic performance in SMEs studied.

\section{IM and SMEs: What are they?}

It is appropriate to define the meaning of two important concepts used in this paper: IM and SMEs. IM has been defined in different ways by different authors. IM implies the establishment of strategic objectives and positioning for inventories ${ }^{[10]}$. IM is the active control program, which allows the management of manufacturing, sales, purchase, and payment ${ }^{[9]}$. However, we define IM as the management of different inventory components (raw materials, work-in-process and finished goods) by properly managing the crucial determinants of inventory levels considering the specific features of SMEs.

\footnotetext{
* Corresponding author. E-mail address: rajeevn@mgmt.iisc.ernet.in.
} 
SMEs have different definitions in different countries. In India definition of SME is of a recent origin. Till 2006 India did not have a definition for medium enterprise, whereas, small scale enterprise was defined under the Industries Development and Regulation (IDR) Act, 1951. The Micro, Small and Medium Enterprises Development (MSMED) Act, 2006 brought out for the first time a definition for a medium scale enterprise along with a revision of small enterprise definition. The concept of tiny enterprise gave way to micro enterprise. According to MSMED Act, 2006, a micro enterprise in manufacturing sector is defined as an enterprise having investment in plant and machinery not exceeding Rs. 2.5 million. A small enterprise is defined as those having investment in plant and machinery more than Rs. 2.5 million and not exceeding Rs. 50 million. A medium enterprise is defined as those having investment in plant and machinery more than Rs. 50 million and not exceeding Rs. 100 million. Thus, in India, all enterprises having investment in plant and machinery up to Rs. 100 million are SMEs. For the present study we define SMEs as those enterprises having a current replacement value of capital up to Rs. 100 million in plant and machinery.

\section{An overview of literature and objectives}

IM performance improvement is considered as one of the most important management functions in the overall context of materials management systems ${ }^{[6]}$. IM enables economic development and makes a significant contribution to the competitive strength of enterprises- large and small- in national as well as international markets ${ }^{[5]}$. However, SMEs generally have not received due attention for the implementation of IM practices with the objective to improve productivity, quality and competitiveness ${ }^{[4]}$. This is evident from the fact that when business strategies are formulated IM is generally not handled as a critical issue ${ }^{[10]}$.

However, the assessment of inventory ordering policies in a small UK based manufacturing enterprise demonstrated a significant potential for inventory cost saving by categorizing its stock ${ }^{[11]}$. Despite such optimism, "IM performance" improvement continues to be a major problem in SMEs. Keeping in mind the benefits that can be obtained from practicing IM; SMEs must treat IM as an important strategic issue. This is further motivated by the fact that SMEs are a major component of industrial sector in both developed and developing economies and many of the inventory-intensive industries operate in the SME sector too.

Even though the benefits of practicing IM such as productivity improvement, improved competitiveness, reduction in processing time and lead time, among others, are discussed by many researchers, previous studies failed to establish any direct relationship between IM and economic performance in SMEs ${ }^{[12]}$. There is hardly any sincere initiative to improve IM performance in Indian SMEs too, in terms of their economic performance. It is with this backdrop that the present study is undertaken with the following objectives in mind.

- To analyze the importance of material and hence IM in SMEs.

- To probe the relationship between IM and economic performance.

\section{Sampling, data collection and methodology}

The study is confined to machine tool industries sector in the city of Bangalore in India. Machine tool industries are rather the most important manufacturing industry concentrated in an around Bangalore ${ }^{[1]}$. It is also considered an inventory intensive industry having formal or informal relationship with various large enterprises including Multi Nationals (MNCs) located in the city. The quality and cost of engineering products depends on the quality of parent machine tools and their automation levels. The development of machine tool industry is therefore of paramount importance for a competitive and self-reliant industrial structure. Therefore, this sector is considered appropriate for our study.

As there is no systematic database of machine tool SMEs located in Bangalore we decided to focus on a maximum number of 100 SMEs in this sector. From these SMEs we gathered primary data through a semi structured questionnaire having five sections on basic features, IM practices and performance, economic variables, production details, factors hindering/ facilitating IM etc. Respondents were asked to gauge the extent to which they agreed with the given statements regarding each question. Mostly items were formulated as short statements and respondents were asked to provide their views on a five point Likert scale. The data 
collection exercise was carried out by the author himself during September 2006 to February 2007. From a total of 100 units covered nine units were eliminated due to incomplete information, inadequacy of data etc. Finally data from 91 SMEs were considered for the final analysis. The methodologies adopted for the data analysis are correlation and regression analysis. The limits of this paper do not allow a detailed evaluation of all survey results. We shall provide the main results and draw some general conclusions.

\section{$5 \quad$ IM and economic performance: results and discussions}

\section{Share of inventory cost and material cost in value of output}

The material cost is the total amount spent on material inputs. The inventory cost is the sum of the ordering cost and carrying $\operatorname{cost}^{[13]}$. However, to appreciate the role of material amongst all inputs in the production process of these SMEs, it is necessary to estimate their production functions. As the production function relates all inputs to the output simultaneously, the effect of variation in one over the other is better reflected by it. Any operation by machine cannot be performed unless enough material is available. The "production function" is essentially an engineering concept that relates the various input factors in production to the output from it. Traditionally, standard production models considered capital, land and labour as the fundamental factors of production. The nature of inputs specified in most present day production models normally consider only capital and labour.

However, 'material' has become a key factor these days in deciding the product cost at micro level as well as in dictating the inflation and the debt burden at the macro level ${ }^{[6]}$. Material cost is a significant factor[8] in economic activity at par with factors of production like capital, land and labour. Therefore, most of the applied economic theories recognize material as an independent factor of production and advocates a KLME (Capital, Labour, Material and Energy) model for economic analyses.

In the present study, material cost share is significant in all SMEs and hence material cost fully deserves to be considered an explanatory factor in production functions. In a similar manner inventory cost is also considered as an independent variable. Though a variety of functional forms are used in economics to describe production, the Cobb-Douglas or multiplicative form is most generally used because it accurately characterizes many production processes ${ }^{[7]}$. Bearing this in mind we have decided to use Cobb-Douglas production function to establish the importance of material and inventory separately amongst the inputs in the SMEs under study. The Cobb-Douglas production function adopted for the analysis is of the form:

$$
Y=A k^{\alpha} L^{\beta} M^{\gamma} E^{\delta}
$$

where:

$Y=$ Value of output of a SME firm in the sector

$K=$ Value of capital (Current value of Plant \& Machinery)

$L=$ Labour cost

$M=$ Raw material and other miscellaneous cost (excluding energy)

$E=$ Energy cost

$A, \alpha, \beta, \gamma$, and $\delta=$ Parameters that when estimated describe the quantitative relationship between the inputs and the output.

Taking logarithms on both sides of equation (1), Cobb-Douglas production function is reduced to a loglinear relationship between output of production and factors of production. Therefore, it essentially takes the form of a multiple regression equation. If all the inputs and output are expressed in monetary terms, the coefficients of independent variables may be used for interpreting the importance of the independent variables in explaining the variation in the dependent variable. Hence, from economics point of view the coefficient of material input indicates the importance of inventory performance in explaining the variation in value of production in the presence of other inputs. The output expressed in terms of its monetary value assists nullifying minor variation, if any, in quality of goods produced by different SMEs in a given industrial sector. We have used the current replacement value of plant $\&$ machinery to represent capital. The current value of 
plant \& machinery in SMEs is obtained from the respective entrepreneurs and it was subsequently confirmed after discussing with the experts in the field.

Economists prefer to express labour in terms of total man-hours or man-days with adjustments for nonproduction workers. However, labour input in the form of man-hours or man-days is extremely difficult to get from SMEs and is also less reliable than labour cost data. This is for the reason that most SMEs do not have any formal payroll and owners are more concerned about labour costs than man-hours. In addition, experts observe that SMEs operate under highly competitive conditions, particularly those which are in the same industry in a sector, such as machine tool industry. In theory, competitive environment leads to equality between marginal productivity and wage rate. Thus, we have used labour cost in place of man-hours or man-days in the analysis. This also suited the statistical requirement of expressing all inputs in the same measuring units to make better comparison of coefficients of independent variables in regression. The material cost is separated out for the obvious reason of finding their exclusive role in explaining the variation in value of output. The production function analysis in SMEs is aimed at answering the question "How important is inventory amongst inputs in explaining variation in the value of output and value added?" This is indicated by the beta coefficients in the multiple regression models shown below. Considering the high percentage share of material cost in the value of output (average value of $42.56 \%$ ), we conducted successive regression by considering material cost and inventory cost separately. The results of the regression analysis are shown below.

Table 1. Production function (regression) analysis (dependent variable: value of output)

\begin{tabular}{c|c}
\hline MACHINE TOOL SMEs & \\
\hline Ln $\{$ Capital $\}$ & $0.140(4.197)[.000]$ \\
Ln $\{$ Labour $\}$ & $0.278(10.132)[0.000]$ \\
LN $\{$ ENERGY $\}$ & $0.096(3.090)[0.003]$ \\
Ln $\{$ Material $\}$ & $0.816(24.419)[.000]$ \\
Ln $\{$ A $\}$ - Const & $1.037(2.377)[0.020]$ \\
Adjusted $R^{2}$ & 0.943 \\
F & $372.472[0.000]$ \\
N & 91 \\
\hline
\end{tabular}

Values within the parentheses and square brackets indicate the ' $t$ ' values and the significance levels respectively.

In the machine tool industry, when all the firms taken together, among the inputs only one significant input found highly useful while explaining the variation in output value (very high value of coefficient). Although capital, labour, and energy consumed has a direct relationship with physical output and it turns out to be significant in explaining the variation in value of output, material input turn distinct from others. Therefore, only one input in the form of materials, is found distinctly significant in explaining the variation in output value of the machine tool SMEs.

We separated out the inventory cost and conducted a regression analysis. The result of the regression analysis is shown in Tab. 2. In the machine tool industry, when all the firms taken together, all inputs found useful while explaining the variation in output value. However, in this case also among other inputs only one input in the form of inventory found highly useful while explaining the variation in output value (very high value of coefficient). Although capital, labour, and energy consumed have a direct relationship with physical output and it turns out to be significant in explaining the variation in value of output, inventory input turned very distinct from others.

Before building the full models as given in Tab. 3, we carried out preliminary regression with material and inventory cost as the only independent variable in each of the three groups (performing, moderately performing and non performing) of enterprises. The regressions got adjusted $R^{2}$ values of as high as 0.880 , 0.949 , and 0.975 respectively when only one input in the form of material was selected as the independent variable. Similarly, the regression got adjusted $R^{2}$ values of as high as $0.707,0.824$, and 0.826 respectively when inventory was selected as the only independent variable in three groups of SMEs. This shows that material and inventory cost is a vital input in explaining the variation in output in all the three groups. Yet, our 
Table 2. Production function (regression) analysis (dependent variable: value of output)

\begin{tabular}{c|c}
\hline MACHINE TOOL SMEs & \\
\hline Ln $\{$ Capital $\}$ & $0.226(4.012)[.000]$ \\
Ln $\{$ Labour $\}$ & $0.181(3.985)[0.000]$ \\
LN $\{$ ENERGY\} & $0.676(12.112)[.000]$ \\
Ln $\{$ Material $\}$ & $0.119(2.235)[0.028]$ \\
Ln $\{$ A $\}$ - Const & $11.774(2.396)[0.019]$ \\
Adjusted $R^{2}$ & 0.833 \\
F & $112.873[0.000]$ \\
$\mathbf{N}$ & 91 \\
\hline Values within the parentheses and square brackets indicate the 't' \\
values and the significance levels respectively. \\
\hline
\end{tabular}

interest is to establish the importance of material cost and inventory cost amongst all the inputs which calls for multiple regression involving all other inputs viz., labour, capital. Multiple regression method with SPSS (Statistical Package for Social Sciences) software is employed for this purpose. The relevant assumptions of regression analysis are validated by performing appropriate statistical tests.

From Tab. 3 and Tab. 4 it can be noted that the regression model is appropriate as they have significant ' $F$ ' values. The independent variables included appear to explain a large amount of variation in the value of output as reflected in high values of adjusted $R^{2}$. But more importantly, material and inventory cost is found to contribute significantly towards explaining the variation in output in all SMEs. It is interesting to note that when material is included as an input along with capital (represented by current value of plant \& machinery), labour and energy all inputs (capital, labour, energy and material) found statistically significant in group 1 SMEs. However, all inputs except material are found statistically insignificant in explaining the variation of the value added in group 2 SMEs whereas, labour and energy found statistically insignificant in group 3 SMEs. Perhaps, this can be attributed to the fact that moderately performing SMEs employ relatively less capital compared to performing SMEs and the material and labour costs override the capital, particularly in the SMEs under study.

Table 3. Production function (regression) analysis (dependent variable: value of output)

\begin{tabular}{l|l|l|l}
\hline \multicolumn{1}{c|}{ SMEs $\rightarrow$} & \multicolumn{1}{c}{ Performing group } & Moderately performing & Non performing SMEs \\
\hline Ln $\{$ Material $\}$ & $0.558(7.085)[0.000]$ & $0.961(13.958)[0.000]$ & $0.857(21.793)[0.000]$ \\
Ln $\{$ Labour $\}$ & $0.185(2.167)[0.043]$ & $0.010(0.184)[0.855]$ & $0.035(0.676)[0.504$ \\
Ln $\{$ Energy $\}$ & $0.186(3.142)[0.005]$ & $0.050(1.092)[0.284]$ & ]$-0.017(-0.525)[0.603]$ \\
Ln $\{$ Capital $\}$ & $0.171(2.628)[0.017]$ & $-0.030(-5.99)[0.554]$ & $0.106(2.777)[0.010]$ \\
Ln $\{\mathbf{A}\}-$ Const & $2.169(1.995)[0.061]$ & $1.935(2.292)[0.030]$ & $1.116(2.454)[0.020]$ \\
Adjusted $R^{2}$ & 0.961 & 0.947 & 0.979 \\
F & $142.305[0.000]$ & $143.757[0.000]$ & $395.054[0.000]$ \\
N & 24 & 33 & 34 \\
\hline \multicolumn{4}{c}{ Values within the parentheses and square brackets indicate the 't' } \\
\hline
\end{tabular}

On the other hand when inventory is included as an input along with capital (represented by current value of plant \& machinery), labour and energy all inputs (capital, labour, energy and material) found statistically significant in group 1 SMEs. However, all inputs except capital are found statistically significant in explaining the variation of the value added in group 2 SMEs whereas, labour and energy found statistically insignificant in group 3 SMEs.

The foregoing results are not surprising because any input to the production process, however small, which bears some fixed relationship with the value added will get significant regression coefficient when the output is regressed on it. But, what is revealing is the magnitude of coefficient of material and inventory cost in the regression models of the SMEs. It indicates the importance of material input or inventory input alone in explaining the variation in value of dependent variable. More interestingly, the proportion of variation in the 
Table 4. Production function (regression) analysis (dependent variable: value of output)

\begin{tabular}{l|l|l|l}
\hline \multicolumn{1}{c|}{ SMEs $\rightarrow$} & \multicolumn{1}{c|}{ Performing group } & Moderately performing & Non performing SMEs \\
\hline Ln $\{$ Inventory $\}$ & $0.344(3.513)[0.002]$ & $0.723(7.041)[0.000]$ & $0.702(7.202)[0.000]$ \\
Ln $\{$ Labour $\}$ & $0.448(4.253)[0.000]$ & $0.179(2.037)[0.051]$ & $0.053(0.553)[0.584]$ \\
Ln $\{$ Energy $\}$ & $0.134(1.532)[0.142]$ & $0.134(1.753)[0.091]$ & $-0.087(-1.016)[0.318]$ \\
Ln $\{$ Capital $\}$ & $0.167(1.618)[0.122]$ & $0.043(0.516)[0.610]$ & $0.323(3.362)[0.002]$ \\
Ln $\{\mathbf{A}\}$ - Const & $5.204(3.808)[0.001]$ & $1.321(0.902)[0.375]$ & $3.144(2.653)[0.013]$ \\
Adjusted $R^{2}$ & 0.914 & 0.848 & 0.872 \\
F & $61.86[0.000]$ & $45.485[0.000]$ & $57.305[0.000]$ \\
$\mathbf{N}$ & 24 & 33 & 34 \\
\hline \multicolumn{3}{|c}{ Values within the parentheses and square brackets indicate the 't' } \\
\hline \multicolumn{3}{c}{ values and the significance levels respectively. } \\
\hline
\end{tabular}

value of output explained by inventory input (beta coefficient) in SMEs appears to be much higher than the respective share of inventory in the value of output in SMEs. This indicates, even if inventory has a modest share in value of output, it accounts for a much higher share of the variation in value added. This further reinforces the importance of inventory as an input in SMEs.

Overall, it can be concluded from the production function analysis that material does make a significant contribution towards explaining the variation of output value in SMEs. In fact, it is found that material is the most important input in the machine tool sector, based on its beta coefficients. IM performance improvement is considered as one of the most important management functions in the overall context of materials management systems $^{[6]}$. In this context, it is meaningful to probe whether IM performance is associated with economic performance in the SMEs.

The average inventory and economic performance obtained through sampled SMEs in each of the groups is presented in Table 5. It may be noted from the table that value addition in performing group is more as reflected by high ITR (i.e. more sales per inventory value). It appears that maximum value addition occurs in enterprises of group 1 followed by group2, and group 3 firms respectively. But, a more useful indicator is value added per value of output, as it represents actual value added share in the unit output value. On this count, also group 1 firms come first followed by group 2 and group 3 firms respectively. For the group 3 firms value added per value of output is lowest reflecting that the labour and capital have very limited role to play in value addition process.

Table 5. Im and economic performance in smes

\begin{tabular}{l|l|l|l}
\hline \multicolumn{1}{c|}{ Indicators } & Group 1 & Group 2 & Group 3 \\
\hline IM performance (ITR) & 9.64 & 3.29 & 1.87 \\
Value Added Per SME (Rs.Lakhs) & 166.67 & 85.23 & 84.78 \\
Value Added Per Value of Output & 0.84 & 0.65 & 0.42 \\
Labour Productivity & 473589 & 335530 & 327743 \\
Capital Productivity & 1.91 & 1.16 & 1.28 \\
Returns to Scale & 1.009 & 0.912 & 0.764 \\
\hline
\end{tabular}

It is apparent that the labour employed is most productive in the performing firms compared to other two groups. As per the definition adopted by us, labour productivity can be high either due to more value addition or due to less labour or both. The group1 is best in terms of labour productivity may be due to its high value addition. While group 3 units are least labour productive largely due to low value addition, group 2 firms are placed in the middle position based on labour productivity.

On the basis of capital productivity, and returns to scale also performing firms fare better than rest of the two groups. It appears that SMEs of group1 utilized the existing capital more effectively in the value addition process. So far, we have discussed IM and economic performance of the three SME groups and broadly compared them. But, what is more significant is to probe how IM performance goes with other economic performance indicators among SMEs within a given sector. Keeping this in mind we conducted a correlation 
analysis suggesting that IM performance is associated with economic performance (Tab. 6). The linkage of IM performance with the economic indicators such as value added, factor productivities (labour and capital), returns to scale is discussed in the following sections.

\section{IM and economic performance}

\subsection{IM performance and value addition}

Gross value added is the difference between value of output and total cost comprising materials and energy. In a given industry sector like machine tool, the value of output from an enterprise depends mostly on quantum of production as the value per-unit of product is more or less equal in the sector. The value added in an enterprise can increase either due to more output value or due to reduced total cost comprising energy and materials. Other things remaining the same, at a given production level if an enterprise is more IM-efficient than the other, then it will have higher value added due to reduced material consumption and wastage. But, the problem with this argument is that value added may also increase due to lower energy consumption excluding material. In addition, with higher production volumes the "economies of scale" is likely to influence the value addition process bringing down the total cost of production not only in material but also in other inputs. In view of this, a strong association between IM performance and total value added by a SME enterprise in a sector is not to be expected. The correlation coefficients obtained substantiate this argument. Though significant, the correlation coefficients are not very high enough to infer that higher IM performance alone leads to more value added in the sector.

A better measure of associating IM performance with value addition would be to consider value added per value of output, as it overcomes the dissimilarities in size of value of output among SMEs. Based on the correlation results (Tab. 6), it can be said that higher IM performance, in other words higher ITR, leads to better value added per value of output as the values of the coefficients are quite high compared to other economic performance variables and also statistically significant. Material being one of the major inputs in all SMEs, its contribution to reduction in total cost and hence increasing the value added is bound to be critical as revealed by these results.

Table 6. Association of im performance with various economic performance indicators

\begin{tabular}{c|c}
\hline Correlation of IM performance (ITR) With & Machine tool SMEs \\
\hline Value Added Per SME & 0.455 \\
Value Added Per Value of Output & 0.751 \\
Labour Productivity & 0.211 \\
Capital Productivity & 0.306 \\
\hline \multicolumn{2}{c}{ All correlations are significant at the 0.01 level } \\
\hline
\end{tabular}

To further reinforce the foregoing argument, we carried out regression analysis as shown in Tab. 7. Since value added per value of output is a measure of how productively a firm employs its factor inputs to maximize its output, we probed the role of IM in this process. Thus, value added per value of output is the dependent variable in the regression analysis with explanatory variables being labour productivity, capital productivity and inventory performance (ITR).

Importantly, ITR is significantly associated with the value added per value of output in all the enterprises along with Capital productivity and labour productivity. This indicates the important role of capital, labour, and IM performance in the value addition process of these SMEs.

\subsection{IM performance and factor productivities}

Factor productivities indicate the efficiency of use of the input factors in the production process. The aim of the correlation analysis is to explore whether SMEs using IM efficiently are also doing the same with 
Table 7. Regression analysis with factor productivities and itr (dependent variable: value added per value of output)

\begin{tabular}{l|c}
\hline \multicolumn{1}{c|}{ SMEs $\rightarrow$} & Machine Tool \\
\hline ITR & $0.245(2.523)[0.013]$ \\
Labour Productivity & $0.245(2.437)[0.017]$ \\
Capital Productivity & $0.184(1.832)[0.070]$ \\
Constant & $0.467(12.363)[0.000]$ \\
Adjusted $R^{2}$ & 0.157 \\
F & $6.590[0.000]$ \\
$\mathbf{N}$ & 91 \\
Values within the parentheses and brackets are the 't' values and significance levels \\
respectively
\end{tabular}

respect to other factors of production. The results of the correlation analysis (Tab. 6) suggest that the IMefficient firms are also efficient in terms of productively using their labour as well as capital. This is logical, as material consumption is more directly connected with capital (plant \& machinery) and labour and hence relationship of ITR with capital and labour is likely to be stronger. Thus, an enterprise having machines \& equipments which are of better quality, better maintained, etc. (as may be reflected by its higher current value of capital) is expected to consume less material per unit of value added. In all SMEs, IM performance is associated with labour and capital as shown in Tab. 6.

\subsection{IM performance and returns to scale in smes}

Another useful dimension of linkage between IM performance and economic performance is to ascertain whether IM performance makes any significant difference to 'returns to scale'. Since 'returns to scale' is a measure of change in output relative to proportionate change in all the inputs including material, a higher 'returns to scale' need not be necessarily due to IM alone. Nevertheless, in view of the importance of material input in the SMEs, enhanced 'returns to scale' appears to be impracticable without improved IM performance. It is in this context that we are probing whether better IM performance leads to any significant enhancement of 'returns to scale' in SMEs. For this purpose, we formed three groups among the sampled SMEs based on their IM performance (ITR). Firms which exhibited more than average ITR in the sector are categorized under "performing" and the remaining under "moderately performing", and "non- performing" groups.

To prove this 'returns to scale' of the three groups (group 1 represents performing enterprises, group 2 represents moderately performing enterprises and group 3 non performing enterprises respectively) in the SME sector is analyzed (Tab. 8). Significant ' $t$ ' values confirm that the three groups within a given sector are distinctly different from each other on IM performance count. It may be noted that among the SMEs the better IM performing ones enjoyed high 'returns to scale' compared to others.

Table 8. Returns to scale in the im efficient groups in smes

\begin{tabular}{c|c|c|c|c|c}
\hline Group & Firms & Mean ITR & 't' Value & Returns to Scale & 'F'Value \\
\hline 1 & 24 & 6.9 & 8.208 & 1.009 & 81.693 \\
\hline 2 & 33 & 3.5 & 9.033 & 0.912 & 18.338 \\
\hline 3 & 34 & 1.9 & 7.466 & 0.764 & 16.331 \\
\hline
\end{tabular}

All 't' and ' $F$ ' values are significant at the 0.00 and 0.01 level respectively

\section{Conclusion}

This paper aimed at analyzing the importance of inventory as an input in the production process. Consequently, the role of IM in improving the economic performance of SMEs is probed from an economic perspective. The estimated production functions confirmed this with beta coefficients of inventory cost ranking first amongst all the inputs. All the economic performance indicators adopted, seem to have a positive 
and significant association with IM performance in the SMEs. On the whole, it appears that SMEs which are IM-efficient are also likely to perform better on the economic front and experience higher 'returns to scale'. Therefore, the SMEs must aim at enhancing their efficiency of inventory use, as it is expected to be associated with multiple benefits.

\section{References}

[1] Peenya industrial association. in: PIA Technical Directory, Peenya, Bangalore, 2003.

[2] G. Flores, X. Wang, T. Burgess. Tuning inventory policy parameters in a small chemical company. Journal of the Operational Research Society, 2003, 54: 350-361.

[3] L. Goonatilake. Inventory management in the manufacturing sector in developing countries. Engineering costs and production economics, 1990, 19: 19-24.

[4] A. Gunasekaran, P.Cecillie. Implementing productivity improvement strategies in a small company. Technovation, 1998, 18(5): 311-320.

[5] B. Lipman. How to Control to Reduce Inventory, 11-28. 2nd edn. Englewood Cliffs, N.J, Prentice-Hall, 2975.

[6] R. Mohanty. Inventory problems under multiple constraints: Some studies. Engineering Costs and Production Economics, 1985, 9: 355-367.

[7] C. Petersen, W. Lewis. Managerial Economics, 4th edn. Prentice Hall, India, New Delhi.

[8] C. Ptak. A comparison of inventory models and carrying costs. Production and inventory management journal, 1988, 29(4): 1-2.

[9] Ricklavely. Can you profit from improved inventory control. Auto Inc Magazine, 1996, XLIV/3.

[10] L. Sprague, J. Wacker. Macroeconomic analyses of inventories: Learning from practice. International Journal of Production Economics, 1996, 45: 231-237.

[11] G. Vastag, D. Whybark. Inventory management: Is there a knock-on effect? International Journal of Production Economics, 2005, 93-94: 129-138.

[12] R. Vergin. An evaluation of inventory turnover in the fortune 500 industrial companies. Production and Inventory Management Journal, 1998, 39(1): 51-56.

[13] A. Zeng. Efficiency of using fill-rate criterion to determine safety stock: A theoretical perspective and a case study. Production and inventory management journal, 2000, 42(2): 41-47. 